\title{
Contenido Nutricional de Líneas de Fríjol Caupí (Vigna unguiculata L. Walp.) Seleccionadas de una Población Criolla
}

\author{
Hermes Araméndiz-Tatis, Carlos E. Cardona-Ayala y Enrique M. Combatt-Caballero \\ Universidad de Córdoba. Departamento de Ingeniería Agronómica y Desarrollo Rural, \\ Carrera 6 No. 76-103, Montería - Córdoba, Colombia, (e-mail: haramendiz@hotmail.com, \\ ccardonaayala@yahoo.es, ecombatt@hotmail.com)
}

Recibido Sep. 16, 2015; Aceptado Nov. 17, 2015; Versión final Dic. 16, 2015, Publicado Abr. 2016

\begin{abstract}
Resumen
El objetivo del estudio fue identificar líneas de fríjol caupí con alto contenido de proteína y minerales, a partir de una población nativa. El fríjol caupí constituye una alternativa nutricional importante en el Caribe colombiano. Se evaluaron 39 líneas de fríjol caupí y cinco testigos, incluyendo la población parental, bajo el diseño completamente al azar con cinco repeticiones. El análisis de varianza reveló variabilidad genética entre los genotipos en relación con los contenidos de proteína, calcio, magnesio y potasio, lo que sugiere la posibilidad de obtener cultivares con ventajas nutricionales para los consumidores. Se identificaron las líneas L-047, L-042, L-026, L-029, L-019 y L-002, cuyos contenidos de proteína superaron al del parental Criollo-Córdoba entre 6,28\% y 8,42\%. Estas líneas promisorias podrían ser usadas, en el corto plazo, como variedades o como fuente de genes en programas de mejoramiento genético orientados a la biofortificación de cultivares de la misma especie.
\end{abstract}

Palabras clave: deficiencias nutricionales; proteína; fósforo; hierro; genotipos de fríjol caupí

\section{Nutritional Content of Cowpea Bean Lines (Vigna unguiculata L. Walp.) Selected from a Creole Population}

\begin{abstract}
The objective of the study was to identify cowpea lines with protein and minerals high content, from one native population. Cowpea bean is an important nutritional alternative in the Colombian Caribbean. Thirty nine cowpea lines and five controls, including the parental population, under completely randomized design with five replications were evaluated. The analysis of variance revealed genetic variability between genotypes, suggesting the possibility of obtaining cultivars with nutritional benefits for consumers. The lines L-047, L-042, L-026, L-029, L-019 and L-002, whose protein content exceeded the parental Criollo-Córdoba between $6.28 \%$ and $8.42 \%$, were identified. These promising lines could be used in the short term, as varieties or as a source of genes in breeding programs oriented to biofortification of cultivars of the same species.
\end{abstract}

Keywords: nutritional deficiencies; protein; phosphorus; iron; cowpea bean genotypes 


\section{INTRODUCCIÓN}

El fríjol caupí (Vigna unguiculata L. (Walp) es una leguminosa anual; originaria de África e India y ampliamente cultivada en áreas tropicales y subtropicales (Carvalho et al., 2012) ) es utilizada como fuente de proteína, calorías, fibra, minerales y vitaminas (Kabas et al.,2007) especialmente en los estratos de bajos ingresos económicos por su bajo costo de producción y acceso económico - social (Sinha y Kawatra, 2003; Santos et al., 2007; Frota et al., 2008); ocupa el segundo lugar después de los cereales como fuente de carbohidratos y proteínas en la dieta humana (Gupta et al.,2010). En 2013, en el mundo fueron cosechadas 11.926.786 hectáreas con rendimiento promedio de $521 \mathrm{~kg} \mathrm{ha}^{-1}$ (FAO, 2013), mientras que en Colombia el área cultivada fue de 14.000 hectáreas con rendimiento promedio de $600 \mathrm{~kg} \mathrm{ha}^{-1}$, especialmente en el Caribe colombiano en pequeñas parcelas que oscilan entre 1.000 y $10.000 \mathrm{~m}^{2}$ (Araméndiz et al., 2011).

De acuerdo a White y Broadley (2009) de los seis billones de habitantes del mundo, el $60 \%$ registra deficiencias en hierro ( $\mathrm{Fe}$ ), $30 \%$ en zinc $(\mathrm{Zn}), 30 \%$ en yodo (I) y $15 \%$ en selenio (Se). Adicionalmente, el calcio $(\mathrm{Ca})$, magnesio $(\mathrm{Mg})$ y cobre $(\mathrm{Cu})$ son deficientes en países desarrollados y en vía de desarrollo (Thacher et al., 2006), lo cual es atribuible a la poca disponibilidad de elementos minerales en el suelo y/o a poca capacidad de las plantas de almacenarlos en sus tejidos, asociado al mismo tiempo con la escasa disponibilidad de alimentos de origen animal (Graham et al., 2007).

En Colombia, las cifras reportadas por el DANE (2012) sobre pobreza $(30,4 \%)$ y pobreza extrema $(12,3 \%)$, en 2010, indican que siguen siendo altas, lo que repercute en la salud de los colombianos, ya que existe una desnutrición crónica y global del $13,2 \%$ y 3,4\%, respectivamente, en niños menores de cinco años; retraso en el crecimiento en un $10 \%$ de la población joven, sobrepeso en el $51,2 \%$ de la población total y anemia en el $8 \%$ de los niños entre 5 y 12 años y, del 7,6\% en las mujeres con edad fértil, lo que constituye un problema de salud pública. La malnutrición por deficiencia de nutrientes y alto nivel de consumo de carbohidratos eleva los niveles de insulina e incrementa la susceptibilidad a diabetes, miopía y otras enfermedades crónicas (Cordain et al., 2002).

La explotación de la variabilidad genética para nutrientes esenciales en la alimentación humana a través de la biotecnología y/o mejoramiento genético convencional, es una estrategia promisoria para mejorar la calidad de los alimentos, lo cual tiene una gran demanda a nivel internacional (Bouis et al., 2003). En este sentido, los programas HarvestPlus o Agrosalud, vienen desarrollando cultivares biofortificados en muchas especies, entre las que se destacan yuca, batata, maíz, arroz, trigo, fríjol común y fríjol caupí, en este último con mayores contenidos de hierro y zinc (Drago et al., 2007; Nutti et al., 2013) y proteína (Sing et al., 2003).

El porcentaje de proteína en estudios realizados en semillas de fríjol caupí oscila entre $21,2 \%$ y $27,9 \%$ Gupta et al., 2010); carbohidratos $52 \mathrm{~g} \cdot 100 \mathrm{~g}^{-1}$, hierro 6,8 $\mathrm{mg} \cdot 100 \mathrm{~g}^{-1}$, manganeso $4,1 \mathrm{mg} \cdot 100 \mathrm{~g}^{-1}$ y fósforo $1,5 \mathrm{mg} \cdot 100 \mathrm{~g}^{-1}$. Así mismo, la calidad nutricional está influenciada por el cultivar y factores antinutricionales, tiempo de almacenamiento y tratamiento térmico (Frota et al., 2008). De esta manera, el mejoramiento genético a través de la biofortificación de cultivos constituye una alternativa que viene ganando espacio cada día más, ya que es económica en razón a que la mitad de los pobres subsisten con US \$2 por día y un $30 \%$ con US \$ 1 por día, y respeta los hábitos alimenticios de las personas (Mayer et al., 2008). El estudio fue realizado con el objetivo de identificar líneas promisorias enriquecidas nutricionalmente en una población criolla de fríjol caupí, para contribuir a reducir los problemas malnutrición en los consumidores del Caribe colombiano.

\section{MATERIALES Y MÉTODOS}

\section{Localización}

Esta investigación se realizó en el año 2012 en el área experimental de la Universidad de Córdoba, localizada a $8^{\circ} 50^{\prime}$ de latitud Norte y $75^{\circ} 49^{\prime}$ de longitud Oeste con una elevación de 20 m.s.n.m., en Montería, Córdoba, Colombia, La precipitación promedio anual es de $1.300 \mathrm{~mm}$, temperatura promedio de 28ㅇ C y 6-7 horas promedio de brillo solar (Palencia et al., 2006). El suelo donde se realizó el experimento presentó textura franco-limosa, $\mathrm{pH}=6,4 ; \mathrm{MO}=1,64 \%$ (Walkley y Black); $\mathrm{P}=17,5 \mathrm{ppm}$ (Bray II); $\mathrm{Ca}^{2+}=13,5$ $\mathrm{mg} / \mathrm{kg} ; \mathrm{Mg}^{2+}=8,0 \mathrm{mg} / \mathrm{kg}$ suelo; $\mathrm{K}^{+}=0,04 \mathrm{cmol} / \mathrm{kg}$ suelo (los tres últimos extraídos con acetato de amonio (1 $\mathrm{mol} / \mathrm{L}$ a $\mathrm{pH}=7$ ) y, posteriormente, determinados por absorción atómica.

\section{Material biológico}

Se utilizaron semillas de 39 líneas de fríjol caupí de color blanco-crema, obtenidas previamente en 2008, a través de selección individual por características agronómicas tales como hábito de crecimiento, número de ramas primarias por planta, número de vainas por planta, longitud de la vaina, número de semillas por vaina y peso de 100 semillas, en un área de $2000 \mathrm{~m}^{2}$ en la población "Criollo-Córdoba" y, posteriormente 
sometidas a evaluación con los testigos Criollo-Tierralta, ICA- Betancí, LCPM-35, IT-86 y el mismo parental Criollo-Córdoba, bajo las mismas condiciones ambientales y de manejo agronómico.

\section{Diseño experimental}

Con las determinaciones del contenido nutricional se realizaron análisis de varianza, pruebas de medias de Tukey al $5 \%$ de probabilidad, contrastes ortogonales: líneas vs testigos y líneas vs población nativa original, con el programa SAS versión 9.2.

\section{Análisis nutricional}

Para el análisis del contenido nutricional se procedió a lavar las semillas con agua desionizada; posteriormente fueron sometidas a secamiento en estufa de circulación forzada (Binder - Kasai, Tuttlingen, Alemania) a $70^{\circ} \mathrm{C}$ por 72 horas. Seguidamente, las semillas de cada cultivar fueron molidas y pasadas por un tamiz de $1 \mathrm{~mm}$ para obtener fracciones más pequeñas y nuevamente se sometieron a un secado total por 12 horas, para luego ser almacenadas en recipientes de plástico herméticamente cerrados.

Se determinó nitrógeno total por el método de Kjeldahl, se utilizó un equipo Buchi K-355 (Flawil - Suiza) y el contenido de proteína se cuantificó multiplicando el contenido de nitrógeno por el factor 6,25 . El contenido de minerales se determinó a partir de una muestra de $1 \mathrm{~g}$ de cada cultivar, sometida a una digestión nítrico perclórica (3:1), a través de un calentamiento gradual hasta una temperatura de $350^{\circ} \mathrm{C}$. La cuantificación se hizo por espectrofotometría de absorción atómica de llama, en un equipo Perkin Elmer 3110 (Norwalk, Connecticut, USA), en tanto que el fósforo por absorción molecular en un Perkin Elmer XLS (Norwalk, Connecticut, USA), de acuerdo a la metodología del IGAC (2006).

\section{Análisis de datos}

Con la información registrada en el laboratorio se realizaron análisis de varianza, contrastes ortogonales líneas vs testigos, y líneas vs población nativa original, pruebas de medias de Tukey al $5 \%$ de probabilidad y se estimaron los errores estándar con el programa SAS versión 9.2.

\section{RESULTADOS Y DISCUSIÓN}

Entre los genotipos estudiados, para la mayoría de los componentes nutricionales se evidenció la existencia de diferencias genéticas, con excepción del zinc. Esta variabilidad estimada por los cuadrados medios del análisis de varianza (Tabla 1), reveló diferencias significativas $(P<0,05)$ y altamente significativas $(P<0,01)$, lo que sugiere acumulación diferencial de los minerales en la semilla y permite la identificación de genotipos de mejor calidad nutricional para su uso como alimento. Estos resultados concuerdan con los reportados por Mesquita et al. (2007); Frota et al. (2008) y Antova et al. (2014), en cultivares de caupí originarios de Brasil, Nigeria y Bulgaria. La estimación de los contrastes líneas vs testigos y líneas vs población original CriolloCórdoba permitió la identificación de genotipos con contenido proteínico superior al de la población original.

Tabla 1: Cuadrados medios del análisis de varianza de los contenidos de nutrientes minerales en fríjol caupí

\begin{tabular}{|c|c|c|c|c|c|c|c|c|}
\hline \multirow[b]{2}{*}{ Fuente de variación } & \multirow[b]{2}{*}{$\mathrm{GL}$} & \multirow{2}{*}{$\begin{array}{c}\text { Proteína } \\
\%\end{array}$} & $\mathrm{Ca}$ & $\mathrm{Mg}$ & $\mathrm{K}$ & $\mathrm{P}$ & $\mathrm{Fe}$ & $\mathrm{Zn}$ \\
\hline & & & \multicolumn{4}{|c|}{$\mathrm{g} \cdot \mathrm{kg}^{-1}$} & \multicolumn{2}{|c|}{$\mathrm{mg} \cdot \mathrm{kg}^{-1}$} \\
\hline Genotipos & 43 & $8,08^{* *}$ & $0,04^{\star \star}$ & $0,07^{\star *}$ & $1,89^{\star *}$ & $0,44^{*}$ & $3588,69^{*}$ & $35,41^{\text {ns }}$ \\
\hline Error & 176 & 1,99 & 0,01 & 0,03 & 1,27 & 0,30 & 2248,84 & 57,33 \\
\hline Líneas vs Testigos & 1 & $25,8^{* *}$ & $0,019^{\text {ns }}$ & $0,166^{\text {ns }}$ & $0,010^{\text {ns }}$ & $4,68^{\text {ns }}$ & $3694,7^{\star *}$ & $79,8^{\text {ns }}$ \\
\hline Líneas vs Criollo-Córdoba & 1 & $10,6^{*}$ & $0,015^{\mathrm{ns}}$ & $1,150^{\text {ns }}$ & $0,016^{\text {ns }}$ & $1,19_{\mathrm{ns}}$ & $3691,6^{*}$ & $44,8^{\text {ns }}$ \\
\hline Media general & & 24,77 & 0,60 & 2,28 & 13,01 & 5,21 & 86,81 & 48,66 \\
\hline C.V. (\%) & & 5,70 & 18,99 & 7,91 & 8,65 & 10,55 & 54,62 & 15,55 \\
\hline
\end{tabular}

El contenido de proteína registró porcentajes que variaron entre $22,05 \pm 0,36 \%$ en la línea L-038 y 26,96 \pm $0,78 \%$ en el cultivar LCPM-35, como se observa en la Tabla 2. Estos valores superan a los registrados por Antova et al. (2014), quienes reportaron valores comprendidos entre $20,1 \%$ y $25,8 \%$ y concuerdan con los encontrados por Henshan (2008). El contraste líneas (L-047, L-042, L-026, L-029, L-019 y L-002) vs población nativa original resultó altamente significativo y permitió la identificación de estas líneas promisorias que superaron a la población nativa original Criollo-Córdoba entre el 6,28\% y 8,42\%, lo cual, desde una perspectiva de consumo, contribuiría significativamente al mejoramiento de la calidad de la alimentación de las personas socio económicamente más vulnerables, respetando a la vez sus hábitos alimenticios, ya que la cocción del fríjol caupí mezclado con arroz constituye un alimento mucho más nutritivo y es una costumbre arraigada en el Caribe colombiano, como lo reportan Mesquita et al. (2007); 
por otra parte, los productos extruidos a base de la mezcla maíz/fríjol caupí, mejoraron significativamente la cantidad y calidad proteica, sin afectar los contenidos de hierro y zinc (Drago et al., 2007). A su vez, por tener éstos materiales semillas de color blanco o crema poseen menor cantidad de polifenoles y resultan más aprovechable nutricionalmente (Kachare et al., 1988), si se tiene en cuenta que para niños entre 1 y 3 años, la demanda es de $1,1 \mathrm{~g}$ proteína $/ \mathrm{kg}$ de peso corporal; en niños de 4 a 13 años, 0,95 g proteína/kg de peso corporal; en adolescentes de 14 a 18 años, 0,85 g proteína/kg de peso corporal y en mayores de 18 años, 0,80 g proteína/kg de peso corporal (Hernández, 2004).

El contenido de fósforo osciló entre $4,63 \pm 0,12 \mathrm{~g} \cdot \mathrm{kg}^{-1}$, en la línea $\mathrm{L}-023$ y 5,68 $\pm 0,17 \mathrm{~g} \cdot \mathrm{kg}^{-1}$ en los cultivares Criollo-Córdoba y L-057 (Tabla 2), cantidades que permiten satisfacer la demanda del ser humano. Estos contenidos de fósforo se encuentran dentro de los rangos reportados por Mesquita et al. (2007) y superan los obtenidos por Frota et al. (2008) en el cultivar BRS-Milênio. El contraste líneas vs población original Criollo-Córdoba resultó no significativo, lo que sugiere similaridad en el contenido de este elemento en los materiales estudiados. El fósforo favorece la calidad biológica de la proteína (Mesquita et al., 2007), es importante en la formación y mantenimiento de huesos, por ser componente de la hidroxiapatita ósea, e influyente en el desarrollo de dientes y secreción de leche materna. Además, su demanda en niños menores de un año es de $100 \mathrm{mg} / \mathrm{d}$, en jóvenes entre 9 y 18 años, de $1.230 \mathrm{mg} / \mathrm{d}$ y de $700 \mathrm{mg} / \mathrm{d}$ a partir de los 19 años. Debe tenerse en cuenta que ha sido establecido para este elemento un nivel máximo de ingestión tolerable de 4-5 g/d (Hernández, 2004).

El contenido de calcio registró una variación entre $0,45 \pm 0,01 \mathrm{~g} \cdot \mathrm{kg}^{-1}$ en la línea $\mathrm{L}-018 \mathrm{y} 0,81 \pm 0,16 \mathrm{~g} \cdot \mathrm{kg}^{-1} \mathrm{en}$ la línea L-029, destacándose los genotipos L-003, L-009, IT86 y L-029, con valores de 0,70 \pm 0,07; 0,71 \pm 0,$07 ; 0,74 \pm 0,04$ y $0,81 \pm 0,16 \mathrm{~g} \cdot \mathrm{kg}^{-1}$, mientras que la población parental Criollo-Córdoba, presentó 0,58 \pm $0,06 \mathrm{~g} \cdot \mathrm{kg}^{-1}$ (Tabla 2). Estas magnitudes son inferiores a las reportadas para el cultivar comercial BRSMilênio, de $140 \mathrm{mg} / 100 \mathrm{~g}$ (Frota et al., 2008). El análisis de varianza presentó diferencias significativas y la prueba de separación de medias de Tukey mostró que sólo la línea L-018 posee contenido de calcio por debajo del resto de las líneas. Los contrastes líneas vs testigos y líneas vs población nativa original resultaron no significativos, lo que sugiere que las líneas evaluadas, excepto la L-018, y los testigos junto con la población nativa original presentaron contenidos de calcio muy similares.

Se ha establecido que las diferencias en el contenido de calcio, posiblemente obedezcan a diferencias genéticas, que pueden influir en la capacidad de formar raíces jóvenes que juegan un rol importante en la absorción y en la acumulación del calcio en la semilla, especialmente en la testa, y, también a la disponibilidad de este elemento en el suelo como lo reportan Ribeiro et al. (2012). Posiblemente, la similitud en la acumulación de calcio en la mayoría de las poblaciones evaluadas esté más bien relacionada con la alta fertilidad del suelo donde se llevó a cabo el experimento, ya que presentó niveles óptimos en los elementos esenciales que permiten satisfacer la demanda nutricional de las plantas, como lo sostienen Zhao y McGrath (2009).

Por otro lado, es importante anotar que las contenidos de calcio reportados en la semilla de fríjol caupí, no satisfacen las necesidades humanas de $1000 \mathrm{mg}$ por día, pero contribuyen significativamente a suplir las demandas a través de las dietas que involucren el fríjol caupí, ya que en los países en vías de desarrollo existe un deficiente aporte de este mineral (White y Broadley, 2009).

El contenido de magnesio osciló entre 2,11 $\pm 0,05 \mathrm{~g} \cdot \mathrm{kg}^{-1}$ en la línea $\mathrm{L}-057$, y 2,57 $\pm 0,09 \mathrm{~g} \cdot \mathrm{kg}^{-1} \mathrm{en} \mathrm{L-034,}$ mientras que la población original Criollo-Córdoba registró 2,37 $\pm 0,07 \mathrm{~g} \cdot \mathrm{kg}^{-1}$ (Tabla 2). Estos valores se encuentran por encima de los rangos indicados por Avanza et al. (2013) en cuatro cultivares de fríjol caupí de origen argentino, que oscilaron entre $0,06 \mathrm{~g} / 100 \mathrm{~g}$ y $0,07 \mathrm{~g} / 100 \mathrm{~g}$. El análisis de varianza mostró diferencias significativas entre las medias del contenido de este elemento y la prueba de separación de medias de Tukey indicó que sólo la línea L-034 superó a la población original Criollo-Córdoba. De manera similar a lo acontecido con el calcio, los contrastes líneas vs testigos y líneas vs población nativa original resultaron no significativos, lo que sugiere que las líneas evaluadas, excepto la L-34, y los testigos junto con la población nativa original presentan contenidos de magnesio semejantes y su absorción en cantidades similares podría estar relacionada con la alta fertilidad del suelo, que constituye una de las estrategias para incrementar el contenido de minerales en los órganos de interés de las plantas (Zhao y McGrath, 2009).

Los contenidos de magnesio reportados para fríjol caupí satisfacen las necesidades del ser humano que están entre los $360 \mathrm{mg}$ y $410 \mathrm{mg}$. Sin embargo, su absorción puede reducirse entre un 20\% y 50\%, por efecto de la cocción (Avanza et al., 2013). De este elemento, es relevante su participación en al menos 300 reacciones enzimáticas, y el efecto protector contra la hipertensión con la consecuente reducción de problemas cardiacos (Rodríguez - Castillo y Fernández - Rojas, 2003). 
Tabla 2: Valores medios y errores estándar de los contenidos nutricionales de 44 genotipos de fríjol caupí (Vigna unguiculata L. (Walp).

\begin{tabular}{|c|c|c|c|c|c|c|c|}
\hline \multirow[b]{2}{*}{ GENOTIPO } & Proteína & $P$ & $\mathrm{Ca}$ & $\mathrm{Mg}$ & $\mathrm{K}$ & $\mathrm{Fe}$ & $\mathrm{Zn}$ \\
\hline & $\%$ & \multicolumn{4}{|c|}{$\mathrm{g} \cdot \mathrm{kg}^{-1}$} & \multicolumn{2}{|c|}{$\mathrm{mg} \cdot \mathrm{kg}^{-1}$} \\
\hline LCPM-35 & $26,90 \pm 0,79$ & $5,65 \pm 0,26$ & $0,58 \pm 0,04$ & $2,16 \pm 0,07$ & $12,82 \pm 0,27$ & $72,97 \pm 19,4$ & $47,24 \pm 2,67$ \\
\hline L-047 & $26,76 \pm 0,73$ & $5,51 \pm 0,33$ & $0,51 \pm 0,07$ & $2,12 \pm 0,08$ & $13,47 \pm 0,56$ & $120,53 \pm 31,1$ & $53,38 \pm 4,84$ \\
\hline L-042 & $26,76 \pm 0,47$ & $5,62 \pm 0,38$ & $0,49 \pm 0,04$ & $2,14 \pm 0,04$ & $13,10 \pm 0,50$ & $177,90 \pm 54,6$ & $49,62 \pm 3,42$ \\
\hline L-026 & $26,48 \pm 0,43$ & $5,55 \pm 0,27$ & $0,63 \pm 0,03$ & $2,30 \pm 0,08$ & $13,18 \pm 0,32$ & $70,70 \pm 13,9$ & $50,58 \pm 3,66$ \\
\hline L-029 & $26,47 \pm 0,77$ & $5,15 \pm 0,09$ & $0,81 \pm 0,16$ & $2,53 \pm 0,12$ & $13,48 \pm 0,51$ & $108,72 \pm 15,2$ & $51,33 \pm 2,97$ \\
\hline L-019 & $26,32 \pm 0,37$ & $5,25 \pm 0,18$ & $0,62 \pm 0,04$ & $2,40 \pm 0,08$ & $13,54 \pm 0,08$ & $106,86 \pm 26,9$ & $50,87 \pm 1,98$ \\
\hline L-002 & $26,23 \pm 0,91$ & $4,99 \pm 0,28$ & $0,67 \pm 0,05$ & $2,24 \pm 0,10$ & $13,20 \pm 0,72$ & $92,85 \pm 22,6$ & $49,74 \pm 3,95$ \\
\hline L-070 & $25,97 \pm 0,42$ & $5,66 \pm 0,24$ & $0,69 \pm 0,05$ & $2,10 \pm 0,04$ & $13,16 \pm 0,40$ & $101,40 \pm 20,2$ & $51,76 \pm 2,92$ \\
\hline L-056 & $25,87 \pm 0,56$ & $5,56 \pm 0,22$ & $0,64 \pm 0,05$ & $2,13 \pm 0,06$ & $13,37 \pm 0,37$ & $86,68 \pm 15,9$ & $51,87 \pm 3,69$ \\
\hline L-057 & $25,84 \pm 0,44$ & $5,68 \pm 0,17$ & $0,58 \pm 0,05$ & $2,11 \pm 0,05$ & $12,91 \pm 0,40$ & $121,56 \pm 31,6$ & $48,54 \pm 3,10$ \\
\hline L-016 & $25,73 \pm 0,70$ & $4,93 \pm 0,22$ & $0,63 \pm 0,04$ & $2,27 \pm 0,07$ & $13,14 \pm 0,36$ & $60,32 \pm 13,9$ & $43,64 \pm 3,66$ \\
\hline L-055 & $25,69 \pm 0,29$ & $5,54 \pm 0,30$ & $0,60 \pm 0,03$ & $2,25 \pm 0,09$ & $12,95 \pm 0,38$ & $79,79 \pm 14,3$ & $50,62 \pm 3,72$ \\
\hline L-030 & $25,66 \pm 0,49$ & $5,44 \pm 0,27$ & $0,66 \pm 0,05$ & $2,18 \pm 0,11$ & $13,08 \pm 0,54$ & $68,62 \pm 12,9$ & $51,90 \pm 2,95$ \\
\hline L-066 & $25,59 \pm 0,56$ & $5,67 \pm 0,22$ & $0,46 \pm 0,04$ & $2,15 \pm 0,08$ & $13,23 \pm 0,41$ & $85,06 \pm 20,9$ & $48,89 \pm 2,55$ \\
\hline L-031 & $25,46 \pm 0,80$ & $5,16 \pm 0,22$ & $0,52 \pm 0,05$ & $2,26 \pm 0,08$ & $12,45 \pm 0,38$ & $65,71 \pm 12,2$ & $49,84 \pm 4,45$ \\
\hline ICA-Betancí & $25,43 \pm 0,20$ & $5,21 \pm 0,18$ & $0,61 \pm 0,03$ & $2,20 \pm 0,05$ & $14,95 \pm 0,42$ & $72,45 \pm 14,0$ & $50,15 \pm 3,77$ \\
\hline L-033 & $25,40 \pm 0,50$ & $5,36 \pm 0,21$ & $0,55 \pm 0,04$ & $2,30 \pm 0,08$ & $13,50 \pm 0,41$ & $61,33 \pm 12,8$ & $47,45 \pm 3,09$ \\
\hline L-006 & $25,40 \pm 0,50$ & $4,87 \pm 0,18$ & $0,67 \pm 0,06$ & $2,51 \pm 0,14$ & $13,23 \pm 0,41$ & $76,69 \pm 12,7$ & $51,81 \pm 6,27$ \\
\hline L-037 & $25,35 \pm 0,62$ & $5,35 \pm 0,30$ & $0,67 \pm 0,05$ & $2,29 \pm 0,10$ & $13,05 \pm 0,17$ & $59,54 \pm 11,1$ & $45,04 \pm 2,63$ \\
\hline L-034 & $25,08 \pm 0,45$ & $4,90 \pm 0,21$ & $0,66 \pm 0,04$ & $2,57 \pm 0,09$ & $13,68 \pm 0,46$ & $100,68 \pm 22,3$ & $52,29 \pm 3,46$ \\
\hline L-036 & $24,85 \pm 0,62$ & $5,34 \pm 0,33$ & $0,54 \pm 0,02$ & $2,21 \pm 0,06$ & $12,27 \pm 0,59$ & $81,92 \pm 18,1$ & $50,61 \pm 2,82$ \\
\hline C-003 & $24,71 \pm 0,59$ & $5,47 \pm 0,32$ & $0,59 \pm 0,06$ & $2,17 \pm 0,08$ & $12,94 \pm 0,40$ & $90,34 \pm 19,5$ & $47,33 \pm 2,78$ \\
\hline L-003 & $24,69 \pm 0,54$ & $5,41 \pm 0,21$ & $0,70 \pm 0,07$ & $2,17 \pm 0,06$ & $11,61 \pm 2,12$ & $74,80 \pm 16,70$ & $48,52 \pm 4,12$ \\
\hline Criollo- Córdoba & $24,68 \pm 0,51$ & $5,68 \pm 0,17$ & $0,58 \pm 0,06$ & $2,37 \pm 0,07$ & $13,41 \pm 0,28$ & $60,99 \pm 16,50$ & $51,74 \pm 3,04$ \\
\hline C-001 & $24,66 \pm 0,38$ & $5,12 \pm 0,24$ & $0,66 \pm 0,00$ & $2,55 \pm 0,10$ & $13,32 \pm 0,29$ & $82,91 \pm 13,0$ & $52,34 \pm 2,42$ \\
\hline L-028 & $24,63 \pm 0,76$ & $5,15 \pm 0,31$ & $0,56 \pm 0,06$ & $2,36 \pm 0,09$ & $12,83 \pm 0,42$ & $62,03 \pm 11,4$ & $47,54 \pm 2,57$ \\
\hline L-022 & $24,63 \pm 1,61$ & $5,00 \pm 0,3$ & $0,47 \pm 0,03$ & $2,28 \pm 0,08$ & $12,44 \pm 0,38$ & $77,62 \pm 15,6$ & $48,48 \pm 2,76$ \\
\hline L-005 & $24,46 \pm 0,52$ & $4,91 \pm 0,11$ & $0,66 \pm 0,06$ & $2,35 \pm 0,09$ & $12,93 \pm 0,17$ & $124,94 \pm 31,0$ & $46,47 \pm 2,76$ \\
\hline L-009 & $24,43 \pm 0,62$ & $5,29 \pm 0,21$ & $0,71 \pm 0,07$ & $2,34 \pm 0,08$ & $12,90 \pm 0,28$ & $74,67 \pm 9,9$ & $51,28 \pm 3,88$ \\
\hline L-020 & $24,18 \pm 0,39$ & $5,28 \pm 0,12$ & $0,48 \pm 0,03$ & $2,29 \pm 0,07$ & $12,85 \pm 0,42$ & $166,44 \pm 45,6$ & $48,15 \pm 2,52$ \\
\hline L-024 & $23,83 \pm 0,34$ & $4,85 \pm 0,08$ & $0,66 \pm 0,04$ & $2,44 \pm 0,04$ & $12,44 \pm 0,27$ & $68,60 \pm 14,4$ & $47,90 \pm 2,01$ \\
\hline L-018 & $23,79 \pm 0,35$ & $4,97 \pm 0,31$ & $0,45 \pm 0,01$ & $2,19 \pm 0,07$ & $12,54 \pm 0,43$ & $81,34 \pm 15,80$ & $46,80 \pm 3,17$ \\
\hline IT86 & $23,72 \pm 0,30$ & $5,08 \pm 0,25$ & $0,74 \pm 0,04$ & $2,39 \pm 0,08$ & $14,13 \pm 0,29$ & $78,63 \pm 15,4$ & $47,20 \pm 2,52$ \\
\hline Criollo-Tierralta & $23,64 \pm 0,41$ & $4,90 \pm 0,2$ & $0,58 \pm 0,04$ & $2,35 \pm 0,10$ & $12,93 \pm 0,29$ & $82,81 \pm 12,3$ & $45,16 \pm 1,58$ \\
\hline L-014 & $23,58 \pm 0,25$ & $5,33 \pm 0,26$ & $0,58 \pm 0,03$ & $2,34 \pm 0,06$ & $12,88 \pm 0,42$ & $108,68 \pm 26,0$ & $52,00 \pm 3,76$ \\
\hline L-011 & $23,54 \pm 0,38$ & $4,86 \pm 0,23$ & $0,56 \pm 0,00$ & $2,39 \pm 0,05$ & $12,47 \pm 0,26$ & $69,68 \pm 15,8$ & $46,83 \pm 3,27$ \\
\hline L-067 & $23,50 \pm 0,53$ & $5,16 \pm 0,25$ & $0,62 \pm 0,02$ & $2,18 \pm 0,06$ & $12,60 \pm 0,27$ & $63,03 \pm 10,24$ & $45,85 \pm 3,58$ \\
\hline L-032 & $23,48 \pm 0,85$ & $5,00 \pm 0,21$ & $0,48 \pm 0,01$ & $2,22 \pm 0,07$ & $11,85 \pm 0,30$ & $69,62 \pm 12,0$ & $44,87 \pm 3,50$ \\
\hline L-001 & $23,41 \pm 0,98$ & $5,11 \pm 0,31$ & $0,51 \pm 0,04$ & $2,31 \pm 0,10$ & $13,96 \pm 0,30$ & $136,35 \pm 39,2$ & $48,09 \pm 3,60$ \\
\hline L-007 & $22,83 \pm 1,23$ & $5,22 \pm 0,26$ & $0,53 \pm 0,03$ & $2,18 \pm 0,05$ & $13,25 \pm 0,46$ & $87,00 \pm 19,1$ & $48,19 \pm 3,90$ \\
\hline L-025 & $22,81 \pm 0,55$ & $4,96 \pm 0,34$ & $0,66 \pm 0,04$ & $2,24 \pm 0,06$ & $12,88 \pm 0,41$ & $63,69 \pm 10,1$ & $46,18 \pm 3,55$ \\
\hline L-023 & $22,61 \pm 0,51$ & $4,63 \pm 0,12$ & $0,46 \pm 0,02$ & $2,35 \pm 0,08$ & $12,58 \pm 0,2$ & $70,88 \pm 16,7$ & $43,91 \pm 2,37$ \\
\hline C-002 & $22,55 \pm 0,73$ & $5,12 \pm 0,24$ & $0,67 \pm 0,05$ & $2,24 \pm 0,10$ & $12,10 \pm 0,32$ & $85,42 \pm 18,86$ & $43,45 \pm 2,12$ \\
\hline L-038 & $22,05 \pm 0,36$ & $4,63 \pm 0,19$ & $0,57 \pm 0,03$ & $2,23 \pm 0,05$ & $12,00 \pm 0,5$ & $65,80 \pm 11,3$ & $45,77 \pm 4,69$ \\
\hline
\end{tabular}


El contenido de potasio fluctuó entre $11,61 \pm 2,12 \mathrm{~g} \cdot \mathrm{kg}^{-1}$ en L-003 y 14,95 $\pm 0,42 \mathrm{~g} \cdot \mathrm{kg}^{-1}$ en ICA Betancí, mientras que la población original Criollo-Córdoba registró $13,41 \pm 0,27 \mathrm{~g}^{\cdot} \mathrm{kg}^{-1}$, valores superiores a los reportados por Avanza et al. (2013), los cuales oscilaron entre 0,34 g/100 g y 0,68 g/100 $\mathrm{g}$ e inferiores a los obtenidos por Mesquita et al. (2007), quienes encontraron valores entre $15,10 \mathrm{~g} \cdot \mathrm{kg}^{-1}$ y $24,80 \mathrm{~g} \cdot \mathrm{kg}^{-1}$. El análisis de varianza mostró diferencias significativas entre las medias del contenido de potasio, sin embargo, la prueba de separación de medias de Tukey indicó que sólo el cultivar Caupí-Betancí resultó superior a los cultivares C-002, L-038, L-032 y L-003. Los contrastes líneas vs testigos y líneas vs población original resultaron no significativos, lo que sugiere que las líneas y testigos evaluados, junto con la población original Criollo-Córdoba, acumularon cantidades de potasio similares y su absorción por las raíces y almacenamiento posterior en las estructuras de la semilla podría estar relacionada con la alta fertilidad del suelo donde se realizó el experimento, el cual presentó niveles adecuados de varios de los cationes del complejo de cambio en la rizosfera del suelo, como ya se indicó anteriormente.

Los contenidos de potasio reportados son importantes en la alimentación humana, especialmente para las personas que practican bastante actividad física, la cual demanda altas concentraciones de este elemento. Asimismo, el potasio reduce el riesgo de presión arterial causada por una dieta elevada en sodio; pero, para personas con problemas con insuficiencia renal, serían más convenientes los genotipos con bajo contenido de potasio (Ribeiro et al., 2012). La demanda de este elemento es de $4,7 \mathrm{~g} / \mathrm{d}$, de acuerdo a Bruuselma et al. (2013).

El hierro registró valores comprendidos entre $59,54 \pm 11,1 \mathrm{mg} \cdot \mathrm{kg}^{-1}$ en el genotipo L-037 y 177,90 $\pm 54,8$ $\mathrm{mg} \cdot \mathrm{kg}^{-1}$ en L-042, mientras que la población original Criollo-Córdoba registró $60,99 \pm 16,7 \mathrm{mg} \cdot \mathrm{kg}^{-1}$, valores superiores a los reportados en 40 variedades de fríjol común por Astudillo y Blair (2008). El contraste líneas vs población original Criollo-Córdoba resultó significativo, sin embargo, los coeficientes de variación fueron altos, lo que dificulta hacer inferencias con mayor precisión acerca de los materiales evaluados. La búsqueda de materiales ricos en este elemento es importante para satisfacer las necesidades humanas, especialmente en regiones con indicadores altos de anemia. Esta deficiencia nutricional, la más extendida en el mundo, genera fatiga, disminución de la productividad y desarrollo cognitivo desbalanceado. Según Hernández (2004), la recomendación nutricional de hierro es de $8 \mathrm{mg} / \mathrm{d}$ para hombres y $18 \mathrm{mg} / \mathrm{d}$ para mujeres premenopáusicas, en tanto que para la mujer embarazada se eleva esta recomendación a $27 \mathrm{mg} / \mathrm{d}$.

En relación con el zinc, el análisis de varianza tampoco acusó diferencias significativas entre los materiales evaluados. El contenido de zinc osciló entre 43,45 $\pm 2,37 \mathrm{mg} \cdot \mathrm{kg}^{-1}$ en la línea C002 y, 53,38 $\pm 4,84 \mathrm{mg} \cdot \mathrm{kg}^{-1}$ en la línea L-047, duplicando los encontrados por Astudillo y Blair (2008), en el fríjol común. La población parental Criollo-Córdoba registró $51,74 \pm 3,04 \mathrm{mg} \mathrm{kg}^{-1}$. Las concentraciones de zinc encontradas son superiores a las reportadas en esta especie por Frota et al. (2008), Soris y Mohan (2011) y Miquilena e Higuera (2012), quienes observaron valores de 1,14 a 4,80 mg/100 g en cultivares de fríjol caupí, pero inferiores a los de Mune et al. (2013), en harina de fríjol caupí. La recomendación nutricional establecida según Hernández (2004) es de $11 \mathrm{mg} / \mathrm{d}$ para hombres y $8 \mathrm{mg} / \mathrm{d}$ para mujeres; en tanto que para niños menores de 12 años, entre $5 \mathrm{mg} / \mathrm{d}$ y $23 \mathrm{mg} / \mathrm{d}$. La ingesta de fríjol caupi permite satisfacer las necesidades de este elemento importante por su papel en varias enzimas, en el mantenimiento de la integridad estructural de las proteínas y en la regulación de la expresión génica.

\section{CONCLUSIONES}

Sobre el análisis y discusión de los resultados del contenido nutricional de la semilla de fríjol caupí, se concluye: 1) existen diferencias genéticas entre los materiales evaluados, en relación con el contenido proteína, calcio, magnesio y potasio; 2) se identificaron las líneas L-047, L-042, L-026, L-029, L-019 y L-002 cuyos contenidos de proteína son superiores al de la población original Criollo-Córdoba, las cuales podrían ser aprovechadas en la obtención de cultivares de fríjol caupí de mejor calidad nutricional; 3) los contenidos de fósforo, calcio, magnesio, potasio y zinc fueron similares a los de la población original Criollo-Córdoba.

\section{AGRADECIMIENTOS}

A la Universidad de Córdoba-Colombia, por la financiación de la investigación.

\section{REFERENCIAS}

Antova, G.; Stoilova, T. y M. Ivanova. Proximate and lipid composition of cowpea (Vigna unguiculata L.) cultivated in Bulgaria, Journal of Food Composition and Analysis: 33 (2), 146-152 (2014)

Araméndiz, T.H.; Espitia, M. y C. Sierra. Comportamiento agronómico de líneas promisorias de fríjol caupí Vigna unguiculata I. Walp., en el valle del Sinú, Temas Agrarios: 16 (2), 9 - 17 (2011) 
Astudillo, C. y Blair, M. Contenido de hierro y cinc en la semilla y su respuesta al nivel de fertilización con fósforo en 40 variedades de fríjol colombiana. Agronomía Colombiana 26 (3) ,471-476 (2008)

Avanza, M.; Acevedo, B.; Chaves, M. y M. Añon. Nutritional and anti-nutritional components of four cowpea varieties under thermal treatments: Principal component analysis, Food Science and Technology: 51 (1), 148 $-157(2013)$

Bouis, H.E;, Chassy, B.M.y J.O. Ochanda. Genetically modified food crops and their potential contribution to human nutrition and food quality. Trends in Food Science and Technology 14 (5-8), 191-209 (2003)

Bruulsema, T.W.; Heffer, P.; Welch, R.M.; Cakmak, I. y K. Moran. Fertilizar los cultivos para mejorar la salud humana: Un estudio científico (en línea: http://www.fertilizer.org/, acceso: 30 de agosto de 2015), International Fertilizer Industry Association, Paris, Francia (2013)

Carvalho, A.F.U.; Mateus, N.; Farias, D.; Brito, L.; Pereira, R.; Viana, M.; Gouveia, S.; Sampaio, S.; Barbosa, M.; Gomes, G.; Morais, S., Costa, C. y F. Freire. Nutritional ranking of 30 Brazilian genotypes of cowpeas including determinationof antioxidant capacity and vitamins, Journal of Food Composition and Analysis: 26 (1), 81-88 (2012)

Cordain, L., Eaton, S.B., Miller, J.B., Lindeberg, S. y C. Jensen. An evolutionary analysis of the aetiology and pathogenesis of juvenile-onset myopia, Acta Ophthalmologica Scandinavica: 80 (2), 125-135 (2002)

DANE (Departamento Administrativo Nacional de Estadística). Pobreza en Colombia (en línea: https://www.dane.gov.co , acceso: 12 de agosto de 2014), Bogotá, Colombia (2012)

Drago, S.R..; González,R.J.; Chel-Guerrero, L. y Valencia, M.E. Evaluación de la Disponibilidad de Minerales en Harinas de Fríjol y en Mezclas de Maíz /Fríjol Extruidas. Información Tecnológica: 18 (1) ,41$46(2007)$

FAO (Organización de las Naciones Unidas para la Alimentación y la Agricultura). Producción de cultivos (en línea: http://faostat.fao.org, acceso: 29 de agosto de 2015, Roma, Italia (2013)

Frota, K.; Soares, R. y J. Arêas. Composição química do feijão caupi (Vigna unguiculata L. Walp), cultivar BRS-Milênio. Ciênc. Tecnol. Aliment.: 28(2), 470-476 (2008)

Graham, R.D.; Welch, R.M.; Saunders, D.A.; Ortiz-Monasterio, I.; Bouis; H.E.; Bonierbale, M.; De Haan, S.; Burgos, G.; Thiele, G. y R. Liria. Nutritious subsistence food Systems, Advances in Agronomy: 92, 1-74 (2007)

Gupta, P.; Singh, R.; Malhotra, S.; Boora, K.S. y H.R. Singal. Characterization of seed storage proteins in high protein genotypes of cowpea [Vigna unguiculata (L.) Walp.], Physiol. Mol. Biol. Plants: 16(1), 53-57 (2010)

Henshan, F.O. Varietal Differences in Physical Characteristics and Proximate Composition of Cowpea (Vigna unguiculata), World Journal of Agricultural Sciences: 4 (3), 302-306 (2008)

Hernández, T.M. Recomendaciones nutricionales para el ser humano: Actualización, Rev Cubana Invest. Biomed.: 23(4), 266-292(2004)

IGAC (Instituto Geográfico Agustín Codazzi). Métodos analíticos del laboratorio de suelos. Subdirección de Agrología, 6a Edición, 450-499, Bogotá, Colombia (2006)

Kabas, O.; Yilmaz, E.; Ozmerzi, A. e I. Akinci. Some physical and nutritional properties of cowpea seed (Vigna sinensis L.), Journal of Food Engineering: 79 (4), 1405-1409 (2007)

Kachare, D.P.; Chavan, J.K. y S.S. Kadam. Nutritional quality of some improved cultivars of cowpea. Plant Foods for Human Nutrition: 38 (2), 155-162 (1988)

Mayer, J. E.; Pfeiffer, W.H. y P. Beyer. Biofortified crops to alleviate micronutrient malnutrition, Current Opinion in Plant Biology: 11(2), 166-170 (2008)

Mesquita, F.; Corrêa, A.; Abreu, C.; Lima, R. y A. Abreu. Linhagens de feijão (Phaseolus vulgaris L.): composição química e digestibilidade protéica, Ciênc. agrotec.: 31 (4), 1114-1121 (2007) 
Miquilena, E. y A. Higuera. Evaluación del contenido de proteína, minerales y perfil de aminoácidos em harinas de Cajanus cajan, Vigna unguiculata y Vigna radiata para su uso en la alimentación humana, Revista Científica UDO Agrícola: 12 (3),730-740 (2012)

Mune, M.A.; Minkaa, M.R. e I.L. Mbombeb. Chemical composition and nutritional evaluation of a cowpea protein concentrate, Global Advanced Research Journal of Food Science and technology: 2(3), 35-43 (2013)

Nutti, M.R.; Carvalho, J.L. V. y E. Watanabe. A biofortificacao como ferramenta para combate a deficiencias em micronutrientes. (en línea:http//www.cprm.gov.br, acceso: 21 de noviembre de 2015), 1-5 (2013)

Palencia, G.; Mercado, T. y E. Combatt. Estudio agroclimático del departamento de Córdoba, 12-126, Ed. Gráficas el Caribe, Montería, Colombia (2006)

Ribeiro, N.D.; Maziero, S.M.; Prigol, M.; Nogueira, C.; Piano, D. y M. Possobom. Mineral concentrations in the embryo and seed coat of common bean cultivars, Journal of Food Composition and Analysis: 26 (1), 8995 (2012)

Rodríguez - Castillo, L. y X. Fernández - Rojas. Los frijoles (Phaseolus vulgaris): Su aporte a la dieta costarricense, Acta méd. costarric.: 45 (3) ,120-125 (2003)

Santos, C.A. y L.C. Santos; Rodríguez, M.A. Melhoramento Genético do Feijão-Caupi na Embrapa SemiÁrido (en línea:

http://www.agencia.cnptia.embrapa.br/Repositorio/melhoramento.genetico.caupi1 000flv5utul02wyiv80kxlb3 6w9k8dko.pdf, acceso: 31 de agosto de 2015), Embrapa Semi Árido, Petrolina, Brasil (2007)

Singh, B.B.; Ajeigbe, H.A.; Tarawali, S.A.; Fernández - Rivera, S. y Abubakar, M. Improving the production and utilization of cowpea as food and fodder. Field Crops Research: 84 (1-2), 169-177 (2003)

Sinha, R. y A. Kawatra, A. Effect of processing on phytic acid and polyphenol contents of cowpeas [Vigna unguiculata (L)Walp], Plant Foods for Human Nutrition: 58 (1), 1-8 (2003)

Soris, P.T. y V.R. Mohan. Chemical analysis and nutritional assessment of two less known pulses of genus Vigna, Tropical and Subtropical Agroecosystems: 14 (2), 473-484 (2011)

White, P.J.; Broadley, M.R. 2009. Biofortification of crops with seven mineral elements often lacking in human diets - iron, zinc, copper, calcium, magnesium, selenium and iodine, New Phytologist: 182 (1), 4984 (2009)

Zhao, F. y McGrath, S. Biofortification and phytoremediation. Current Opinion in Plant Biology: 12 (3), $373-$ $380(2009)$ 